\title{
Interdisciplinary process in the field of energy and environment
}

\author{
MIHAELA POPESCU \\ Transylvania University of Brasov \\ 29, Eroilor blvd., Brasov \\ ROMANIA \\ mvp@unitbv.ro
}

\begin{abstract}
The paper presents the results of a interdisciplinary research study of terminology in the field of energy and environment. Some modalities of acquiring terminology in environment and energy field by means of lexical and semantic entities to be mastered in a communication context are presented. Within the framework of a theoretical approach on the communication process, its cultural and social implications, the study is focused on a effects of applying a modern yet traditional learning method, problem-based learning and its benefits for the way information is rigorously organized and the importance of creating maps of representations of knowledge in this precise field. The knowledge-rich contexts contained in the corpora, the semantic relations comprised in the lexical networks are also described.
\end{abstract}

Key-Words: - communication process, maps of representations, terminology, energy and environment, knowledge-rich contexts, problem-based learning, lexical network, terminological structures.

Received: May 24, 2020. Revised: June 30, 2020. 2nd Revised: August 17, 2020.

Accepted: August 25, 2020. Published: August 26, 2020.

\section{Introduction}

This paper is an analysis of the results of a research study conducted within a group of students from the Faculty of Electrical Engineering. The courses and the workshops are held in English the reason for which terminology in this language is required. A questionnaire [1] was designed whose main purpose was that of distinguishing the students'needs. Some of their main concerns were: to master the terminology of the domain, by using different methods of term extraction traditional and modern ones - to search and select the better definitions, to analyse and solve terminological problems, to communicate with specialists within projects and practical placements; to create terminologies in terms of term formation. One student's concern was related to the formation of terms, the etymology helping them to find the origins of the scientific discoveries and finding a way of reconstructing the world we live in. On the other hand, they are interested in knowing at what extent they have to trust dictionaries and how to choose the dictionaries and the glossaries responding to their scientific needs. To create a terminology database in terms of term bank and to use computers in terms of text processing, to adapt themselves to different registers of language, attention being paid to the formal aspect of language, the property of words and the academic style of speaking and writing, to know how to organize information, how to write academic reports for the evaluation of the ordinary examinations or for different academic events are the students main concern. [5].
This interdisciplinary study is devoted to the analysis of the way a connection between terminology and environment and energy field is achieved in order to fulfill the conditions imposed by the contents of the domain and the creation of trainees and students' competence skills in communicating knowledge through using a rich-knowledge documents contained in corpora. The understanding of communication is the way we transmit knowledge in our scientific repertoire for making sense of and interacting with the world.

\section{Communication process - a theoretical approach}

Communication is conceived as a complex ritualized experience in which the term of "meaning" or significance is constituted by an intricate, contested collaboration among institutional, ideological and cultural forces, aiming at creating a fruitful dialogue between recent strains in acquiring terminology in the environment and energy domains and the ways the older forms of acquisition and the more modern methods are committed to a discourse that is intellectually rigorous yet accessible.

Man lives in a new dimension of reality that is symbolically represented. Communication, even the scientific communication with its rigorous structure rules, is the primary phenomenon of experience. The simplest expression of it is that of a map; where lines, angles, names, squared forms transform the vacant space into a featured environment. Then, this space is 
understood and manageable when it becomes represented in symbolic forms. The map stands for a representation of an environment capable of clarifying a problematic situation. It is able of guiding behavior and transforming undifferentiated space into configured space - known, apprehended and understood [2].

Environment, any given space can be mapped in different modes - artistic, visual, architectural, verbal - and like ordinary language; each mode allows one to speak about or represent something, when the thing in question is not present. This capacity of displacement leads to the production of a complicated act when the "real" stimulus is not present. The absence is replaced by the presence of symbols. These symbolic forms are productive and the learner is able of producing an infinite number of representations, using visual, auditive or language symbols in an infinite set of sentences.

This map represents a simplification of or an abstraction form of the environment. Space is made manageable by the reduction of information. Different maps bring the environment alive in various ways: they produce different realities as maps constitute nature itself. An implication concerns the nature of the thought. This thought is not a private one, but it is public and social. Thinking consists of building maps of environments.

To study communication involves examining the construction, apprehension and use of models of communication themselves- their historical creation and use - in encounters between producer and consumer, teacher and student. Within these encounters lie models of human contact and interaction.

The models of communication create what we pretend they describe. Science is a reflexive one. We do not describe behaviour, we create a particular corner of culture that determines the kind of communicative world we inhabit, as Alvin Gouldner states [2]. In fact, world is partly made up of clichés, verbal of another kind. Our existing model of communication is less then an analysis than a contribution to the chaos of modern culture. Education is thought in terms of potentiality for economics and politics. Thought, modeled within the education process, is the construction and utilization of these maps, models or templates or other any name we convey.

To study communication is to examine the social process wherein significant symbolic forms are created, understood and used. Our attempt to construct, maintain, repair and transform reality is a public activity and it would be of much consequence the words of Raymond Williams: "Communication begins in the struggle to learn and to describe. To start this process in our minds and to pass on its results to the others, we depend on certain communication models, certain rules or conventions through which we can make contact. We can change these models when they become inadequate or we can modify and extend them. Our efforts to do so, and to use the existing models successfully, take up a large part of our living energy [...]. Moreover, many of our communication models become, themselves, social institutions. Certain attitudes to others, certain forms of address, certain tones and styles become embodied in institutions that are then very powerful in social effect [...]. These arguable assumptions are often embodied in solid, practical institutions that then teach the models from which they start". [2]

Under these circumstances, the models of communication are not representations of communication, but representations for communication: templates that guides, concrete processed of human interactions. Recasting our studies of communication in terms of a ritual model is to give us a way in which to rebuild a model of and for communication of some restorative value in reshaping the common culture.

John Dewey, the famous American thinker opens a chapter in Experience and Nature with the claim that "of all things communication is the most wonderful" [2] and completed afterward by the following comment: " Society exists not only by transmission, by communication, but it may fairly be said to exist in transmission, in communication"[2]. The power of his works derives from his working over the views of communication. It is the most wonderful thing because it is the basis of the human fellowship: it produces the social bonds, ties men together and makes associated life possible. Society is possible because of the binding forces of shared information circulating in an organic system. Paraphrasing the American thinker, we can assert that communication is the way in which men come to possess things in common - aims, beliefs, aspirations, knowledge, a common understanding. This consensus demandes communication.

The commonest in all industrial cultures is the transmission view of communication that dominates the dictionary entries and is defined by terms such as "imparting", "sending", "transmitting" or "giving information to others". The center of the idea of communication by transmission of signals or messages over distance for the purpose of control, derives actually from the most ancient of human dreams, that is the desire to increase the speed and effect of messages as trey travel in space. From the time upper and lower Egypt were unified under the First Dynasty until the invention of the telegraph, and then transportation and communication were inseparably linked. Our orientation to communication remains a basic one, at the deepest roots of our thinking, in the idea of transmission: 
communication is a process whereby messages are transmitted and distributed in space for the control of distance and people.

The American economist of the $19^{\text {th }}$ century, Henry Charles Carey, founding member of the Society for the Promotion of National industry of Pennsylvania, in his Unity of Law, [2] substituted the language of electricity for the language of mechanics, identifying the physical laws of electricity and magnetism, then being discovered, with the laws of society and projecting electricity as the new bond between nature and society: "Electricity presents a far more striking resemblance to the brain power which is its correspondent in the societary life [...]. The actual relation of each, and every member of a community as giver and receiver, teacher and learner, producer and consumer is positive and negative by turns and relatively to every difference of function and force in his associates, the whole mass constituting a great electric battery to which each individual contributes his pair of plates. Perfect circulation being established as a consequence of perfect development of all individualities, the economic force flows smoothly through every member of the body politic, general happiness and prosperity [...]. Everywhere in the ratio in which each and every pair of plated is placed in proper relation with each other; the vitalized circuit being thus established throughout the entire mass and made to bear, with the concentrated energy of the whole upon every object of general interest [...]. The more the power is exercised in the direction of promoting rapid circulation, among the plates of which the great battery is composed, the greater is the tendency to the development of an inspiration and an energy closely resembling the service of the lightning of heaven subdued to human use"[2]

From now on, electricity and communication become partner concepts and they acquire the monopole and one can speak about the universal and real democracy of technology. And all this is due to one of the major inventions in the world of science, the innovation of the telegraph that can stand metaphorically for all the innovations that ushered in the modern phase of history and determined the major lines of development of communication [2] The telegraph brought about changes in the nature of language, of ordinary technology, of the very structures of awareness. The telegraph is not seen as a toy, prefiguring the computer, but a demonic device dissipating the energy of history.

\section{Terminology and communication}

This emerging intellectual field, as terminology is, aims at moving beyond the inherited conceptions upon a simple catalogue of nouns specific for the domain, in other terms named, in latin, the "nomenclatura", by recognizing the complexity and diversity of terminology in a communicative context, wherein the two concepts are embedded.

The students, as future experts, are not expected to work as terminologists do (i.e., extracting terms, analyzing them in terms of semantic relations), instead they should learn how to analyze the field they work in, to structure knowledge and to map hypertexts, to avoid illogical representations, to design database structures; to use unambiguous expressions, to use an economical, but expressive style. Starting from the statement that students live in a world dominated by technologies, they are taught to be able to understand and to explain complex technical facts; to use graphical presentations for system structures; to select the correct terminology; to develop terminology together with the product designer and others involved in product development; to communicate with different groups of specialists and to understand technical documentation, documentation systems and structured texts. They have to become aware of the difference between everyday language and special language, to be more accurate with special terminologies, concepts and definitions, to distinguish the principles, to analyze unfamiliar subject field, to think systematically in order to be able to structure texts, hypertexts and other presentations with the help of computers and to make up original papers containing the results of their research as to be read within international conferences or professional meetings and submitted to critics and judgment of the "connaisseurs" in the subject field.

A term is considered as the linguistic label of a concept as mental representation, interpreted in its traditional meaning, and the classical doctrine of terminology relies on a unifying view of knowledge starting from the fact that knowledge is organized into domains and each domain is equivalent to a network of concepts [7].

Linguists and terminology researchers focused their attention on the notion of contexts rich in knowledge (knowledge-rich contexts) that are involved in the detection of terms and properties of terms. Some others, use textual contexts to identify textual segments with semantic relations between them. "From the point of view of terminology, therefore, the lexicon of a language consists of the many separate subsystems representing the knowledge structure of each subject field or discipline. Each knowledge structure consists of variously interlinked concepts" [7] that can be defined as lexical networks.

The semantic relations are everywhere present within the lexical network [4] whose aim is to relate terms to each 
other via a synset - a group of words with the same part of speech that can be interchanged in a certain context. A possibility to master the mechanism of synsets is that of solving problems in acquiring terminology

\section{Solving terminological problems in energy-environment field}

Problem-based learning aiming at acquiring terms and terminological structure in the field of environment and energy is one of the most benefic interactive methods one can adopt.

It is an approach that seeks to open new analytical possibilities by finding new kinds of data and asking new questions and to propose new theories, by analyzing patterns of communication as part of scientific knowledge and behavior. Thus, it entails recognition of both the diversity of communicative possibilities and practices and the fact that such practices are an integrated part of a particular field of science.

Interactive methods are used as alternative modes of teaching to the traditional ones. In this case, some variables must be considered: a) input variables: the quality of learning and motivation relying on different environment learning aspects - e.g., authenticity, social and academic support, competitive spirit allowing autonomy in the process of learning; motivation of studying given by temporal and instrumental prospect, feeling of identification with the institution's personality, choice of goals and types of study; cognitive and behavioral engagement within the learning process; b) output variables: satisfaction and interest depending on the content of studies and the effect expecting to attain.

For acquiring communication skills in manipulating the particular terminology in the field of environment and energy, problem-based learning is, an interactive method that already has demonstrated its potential and importance [5]. A learner-centered method of instruction, skills of cooperation, critical thinking and self-direction are trained to acquire performance in the following cases: appreciation of the diversity of inputs coming from different members of the group; a better management of time, higher capacity in retrieving information, by using libraries and the Internet resources, ability in communicating information by writing reports, or making seminar presentations. A fact that must not be neglected is that a problem acts as a focus of learning and allows the integration of concepts, terms and terminological structures in a complex system for solving the problem. Thus, the communication process is more effective within a group, without forgetting the importance of self-learning.

Our experimental research conducted among the group of students from the Faculty of Electrical Engineering and the projects run within the European framework had those objectives targeted at modeling the environmental map: developing skills to identify a problem and designing appropriate solutions; developing an ability to identify issues that secure further discussions and self-study within the context or content of the problem and providing the skills necessary to become a self-directed learner; functioning as an active participant in the team work engaged in learning and designing an engineering solution; physical, social and environment factors are taken into account when planning the process of solving the problem; developing personal behavior and attitudes - responsibility and dependability, ability to work and communicate in and outside the group, ability to evaluate the personal progress of the other members of the group and of the entire group.

\section{Conclusions}

Studying terminology regardless of the domain is an act of culture. It helps us to map our environment, to furnish it and to create realities, even if they may be dreams, and to give it energy to live. It is a public and social activity. It implies the will and power of building worlds. The merely desire to seek the origins of term formation in a certain domain, eternal quest of identifying and recognizing the world means the recreation of the entire adventure of mankind of knowing, understanding and naming the world in which we live. Knowing the past, one master the future. And this world once known is always raising problems that men are supposed to solve.

\section{References:}

[1] Schiffrin, D., Lexical Acquisition. Approach to Discourse, Blackwell Oxford UK and Cambridge USA, 1994.

[2] Carey, W. J., Communication as Culture. Essays on Media and Society, Routledge, New York, London, 1989.

[3] L'HOMME M. C., Fonctions lexicales pour représenter les relations sémantiques entre les termes, in Traitement automatique des langues (TAL), 43(1), 2002

[4] L'HOMME M. C.,, La terminologie: principes et techniques, Les Presses de l'Université de Montréal, Canada, 2004, pp. 98-101.

[5] Popescu M., Research on Terminological Concept Systems as Knowledge Modelling, in CHER 2009 Conference, Sozopol, Heron Press, Sofia, 2009, p. 425426.

[6] Popescu, M., Defining Semantic Relations in the Domain of Traffic Engineering: Urban Transport in $3^{\text {rd }}$ WSEAS International Conference on Urban Planning and Transportation (UPT'10), Corfu Island, July 22-24,

[7] Sager, J. C., , A Practical Course in Terminology Processing,John Benjamins, Philadelphie/Amsterdam, 1990, p. 13. 


\section{Creative Commons Attribution License 4.0} (Attribution 4.0 International, CC BY 4.0)

This article is published under the terms of the Creative Commons Attribution License 4.0

https://creativecommons.org/licenses/by/4.0/deed.en_US 